\title{
Beiträge zur Klinik und Histologie des Granuloma annulare.
}

\author{
Von \\ Dr. O. Grütz und Dr. E. Hornemann. \\ (Aus der Dermatologischen Universitätsklinik in Kiel [Direktor: Prof. \\ Dr. Kling müller].)
}

Mit 2 Textabbildungen.

(Eingegangen am 17. März 1921).

Der erste Fall von Granuloma annulare ist von Colcot Fox im Jahre 1895 in „Brit. journal of dermatol." unter dem Namen „Ringed eruption“ beschrieben worden. Nach 7 Jahren veröffentlichte Radcliffe - Crocker im selben Journal 4 Fälle und prägte als Erster den Namen „Granuloma annulare“. Erst allmählich hat dieser Name die übrigen verdrängt und ist jetzt der übliche geworden, nachdem in der Literatur eine Menge verschiedener Namen für offenbar dieselbe Affektion ziemliche Unklarheit verursacht hatten. Diese Namen sind: Ringed eruption, Erythema elevatum et diutinum, Eruption circinée chronique de la main, Lichen annularis, Tumores benigni sarcoides cutis, Erythemato-scléroses circinées du dos des mains, Neoplasie nodulaire et circinée des extremités, Sclérose circinée des doigts, Akanthoma annulare, Stéréo-phlogose nodulaire et circinée, Celluloma annulare.

Aus Deutschland sind bis jetzt nur 7 Fälle von Granuloma annulare bekannt geworden, und zwar veröffentlichten Galewsky, Halb, Arndt, Zweig je 1 Fall, und Blaseh ko stellte 2 Fälle in der Berl. Derm. Ges. und Arning 1 Fall in der Hambg. Derm. Ges. vor. In Deutschland publizierte Arndt 1911 die erste größere und kritische Arbeit. Nach ihm veröffentlichte 1914 Kla ussner aus der Prager Klinik und 1916 Liebreich aus der Berner Klinik je eine Arbeit. Man ersieht aus dem Angeführten, wie spärlich die Berichte aus Deutschland sind. Aus allen anderen Ländern ist über die Krankheit mehr veröffentlicht worden; besonders hervorzuheben ist die Monographie von Graham Little, die 1908 in „Brit. journal of. dermatol.“ erschien, welche die ersten 49 Fälle der Weltliteratur enthielt. Erwähnt sei noch, daß in keinem der jetzt gangbaren deutschen Lehrbücher über Hautkrankheiten das annuläre Granulom behandelt wird; nur das Lehrbuch von Pinkus macht hier eine Ausnahme.

Das klinische Bild des typischen Granuloma annulare steht jetzt fest. Es handelt sich klinisch um das Auftreten von nicht schmerzhaften, nicht juckenden, weißlichen, keloidartigen cutanen Knötchen, die fast nur Handrücken und Finger befallen. Diese Knötchen bilden späterhin eine periphere erhabene Randzone mit einem eingesunkenen Zentrum. Die Krankheit besteht Monate bis Jabre. Oft verschwinden die alten, und neue Efflorescenzen treten auf. Befallen werden können beide Gesehlechter, in jedem Lebensalter. Die erkrankten Menschen sind sonst gesund. 
Histologisch sind die Befunde der verschiedenen Autoren nicht ganz übereinstimmend, größtenteils handelt es sich nm eine circumscripte Anhäufung von epitheloiden Zellen in den tiefen Schichten der Haut und Auftreten vom Lymphocyten in deren Peripherie. In der Mitte dieser Infiltrate ist Degeneration oder Nekrose des kollagenen Bindegewebes vorhanden. Die Gefäße sind vermehrt, $\mathrm{nm}$ sie herum sind lymphocytäre Infiltrate zu bemerken. Die Epidermis ist im wesentlichen unverändert.

Über die Ätiologie des annulären Granuloms existieren bis jetzt nur Vermutungen. Einige davon, wie die Zugehörigkeit zu den Tuberkuliden (Little) oder zu den Boeckschen Sarkoiden (Rasch und Gregersen, Galewsky) sind bald verlassen worden. Auch Zieler in Jesioneks "Ergebnisse der Haut- und Geschlechtskrankheiten " und Lewandowsky in der "Tuberkulose der Haut" lehnen eine Zugehörigkeit zu den Tuberkuliden ab. Dalla Faverna sondert einen Teil der Fälle als zu dem ,Erythema elevatum et diutinum" gehörig ab, dem er eine klinische Sonderstellung einräumt. Kla us ner erwähnt das Zusammentreffen von Diabetes mit dem annulären Granulom in 2 Fällen. Liebreich hält eine Verwandtschaft mit dem Lichen ruber planus nicht für ausgeschlossen. Am verbreitetsten ist die Ansicht Arndts, da B das Granuloma annulare zu den spezifischen chronischen Infektionskrankheiten der Haut, deren Erreger z. Z. noch unbekannt ist, gehört.

Auch in der Therapie dieser Krankheit herrscht noch keine Einheit. Innerlich Arsen, dann Pflasterapplikationen, Röntgen, Mesothorium werden verschiedentlich empfohlen. Spontane Ausheilung kommt oft vor.

Da in der Kieler Klinik seit 1908 bis 19207 Fälle von Granuloma annulare zur Beobachtung gekommen, fast alle histologisch untersucht worden sind und von allen gute Lichtbilder existieren, auf deren Wiedergabe hier aber leider verzichtet werden muß, läßt sich an Hand dieser Fälle ein guter Ủberblick über diese seltene Affektion geben.

Fall 1. Charlotte M.

Befallen sind rechter Handrücken und das Dorsum aller Finger, mit alleiniger Ausnahme des 4. Fingers. Vorhanden ist reichliche Knotenbildung. Auf dem Daumen, Zeigefinger und Mittelfinger hat sich ein typischer Ring mit eingesunkenem Zentrum ausgebildet; auf dem Handrücken ist ein solcher Ring anscheinend im Entstehen begriffen.

Histologisch wurde nicht untersucht.

Fall 2. Hedwig B. 15 Jahre alt, Haustochter.

Auf dem rechten Handrücken ist ein Herd z. T. aus einzelstehenden, z. T. aus zusammengeflossenen Knoten gebildet. Auf der Streckseite des linken Zeigefingers ist ein typisch ausgebildeter Herd.

Histologische Untersuchung fehlt.

Fall 3. Frau Dora P. 27 Jahre alt, Hausfrau.

Familienanamnese ohne Besonderheiten.

Vor ungefähr 1/2 Jahr erkrankte Pat. mit kleinen Knötchen an dem Mittel. finger der linken Hand. Seit dieser Zeit erkrankten die Finger bis zur jetzigen Ausdehnung. Frei sind der linke Daumen, der rechte III. und IV. Finger, desgleichen völlig frei sind die Handinnenflüchen.

Status: Am stärksten erkrankt ist das Dorsum der Interphalangealgelenke des II., III. and V. Fingers der linken Hand. In annulärer, stellenweise serpiginöser Gruppierung ist die Haut mit etwas erhabenen, sehr derben, weißlich schimmernden Knoten bedeckt; die Knoten sind stellenweise erbsengroß. Der 
Rand der runden Knoten ist sehr derb und weiß, während das Zentrum etwas eingefallen ist. Über dem 1. Interphalangealgelenk ist ein isolierter, über erbsengroßer, derber, rötlich schimmernder Knoten. An der Daumenseite des rechten Zeigefingers sind die Knoten etwas weniger erhaben und haben eine ovale Form. Auf dem kleinen Finger sind zwei eng aneinanderliegende Knoten. Die Knoten an dem rechten kleinen Finger werden excidiert.

Am 11. X. 1910; 4. XI. 1910; 8. XII. 1910 mit Röntgenstrahlen behandelt und Heilung der Krankheit mit der dritten Bestrahlung erzielt.

22. XI. 1920. Pat. stellt sich wieder vor und macht folgende Angaben: Thre Hautkrankheit sei mit der dritten Röntgenbestrahlung geheilt und nie wieder gekommen. Es wird in Erfahrung gebracht, daß Pat. 1916/1917 wegen einer eitrigen Osteomyelitis am Unterschenkel operiert worden ist; gleichzeitig machte Pat. einen Parathyphus durch. Während dieser Krankheitszeit zeigte Pat. deutliche Erscheinungen der Hysterie. Der jetzt vorgenommene Status ergibt normalen Befund, nur sind noch Reste der abgeheilten Efflorescenzen an der veränderten Hautfelderung zu erkennen.

Im Urin kein Eiweiß, auch kein Zucker.

Im histol og is chen Bild fallen bei der Übersicht mit schwacher VergröBerung zunächst ein großes Infiltrat in der Cutis, perivasculäre Infiltration der Cutisgefäße und auch einige Veränderungen der Epidermis auf. Das Infiltrat besteht aus einem länglich runden septierten Herd, der von der subpapillären Schicht bis fast zur Subcutis in die Tiefe hinabreicht und aus einer sehr zellreichen peripheren ringförmigen Randzone und einem ausgedehnten sehr zellarmen, offenbar nekrotischen zentralen Anteil besteht. Die darüberliegende Epidermis zeigt ein im Vergleich zu der Nachbarschaft erheblich verbreitertes Stratum corneum mit starker Parakeratose. Das Stratum granulosum fehlt an diesen Stellen vollständig. Die Parakeratose besteht nur in dem kleinen Abschnitt, der direkt über dem Infiltrationsherd der Cutis liegt; dementsprechend fehlt das Stratum granulosum nur auf einer kurzen Strecke, tritt in der Nachbarschaft dann sogar in verbreiter. ter und eleidinreicher Schicht auf, die erst in größerer Entfernung vom Krank. heitsherd wieder normale Verhältnisse annimmt. Das Stratum spinosum ist verbreitert und dort, wo die Parakeratose besteht, von spärlichen Wanderzellen durchsetzt. In den basalen, aber auch in den mittleren Zellreihen des Epithels sieht man ungewöhnlich zahlreiche Mitosen. Im übrigen ist eine deutliche Akanthose vorhanden und ein Ödem der papillaren und subpapillaren Schicht. Die Papillargefäße zeigen in der Umgebung eine mehr oder weniger ausgesprochene kleinzellige Infiltration, in der subpapillaren Schicht, in der eine Vermehrung der fixen Bindegewebszellen zu konstatieren ist, wird das Infiltrat erheblich stärker und verdichtet sich in der Richtung nach dem Nekrosenherd immer mehr, um scharf an demselben haltzumachen, bzw. ihn ringförmig von allen Seiten zu umschließen. Das zellige Infiltrat besteht peripher in der Hauptsache aus Lymphocyten, Leukocyten sind ziemlich spärlich vertreten. Nach der nekrotischen Partie ist es immer dichter mit einem Zelltyp untermischt, der nach seiner spindeligen bzw. polygonalen Form, dem großen blasigen Kern und nach seiner blassen Färbung als epitheloider Typ anzusprechen ist. An der Grenze nach dem zentralen nekrotischen Anteil ist die Epitheloidzellenanhäufung am dichtesten. Ganz unvermittelt und scharf abgesetzt beginnt dann der große zentrale nekrotische Herd, in dem die Struktur des Bindegewebes nur schlecht oder überhaupt nicht mehr kenntlich ist und nur spärliche kaum noch färbbare Zellkerne und versprengte amorphe Chromatinpartikel liegen, die als Zelltrümmer anzusehen sind. Von der epitheloiden Randzone ausgehend beobachtet man an einigen Stellen Gruppen von dicht gedrängt nebeneinanderliegenden spindelförmigen Zellen, die zungenförmig in das 
nekrotische Gewebe hineinstreben; die Kerne dieser Zellen sind besonders gut und intensiv gefärbt. Das Bild erweckt den Eindruck einer regenerativen fibroblastischen Tätigkeit, worauf auch gelegentlich sichtbare feine zarte, aber zum Unterschied von der nekrotischen Umgebung gut färbbare, offenbar neu gebildete Bindegewebsfibrillen hindeuten. - Die Gefäße in der peripheren Zone des Erkrankungsherdes sind etwas erweitert und zeigen alle eine ausgesprochene kleinzellige Infiltration, die das Gefäß mantelartig einhüllt. Das Endothel der Intima mancher kleinen Venen ist deutlich hyperplastisch, typische endarteritische Bilder sieht man jedoch nicht. - Die elastischen Fasern sind ebenso wie das kollagene Gewebe überall außer im nekrotischen Herd wohl exhalten, wenn auch durch die Infiltrate vielfach verdrängt. Im Nekrosenherd sieht man nur einzelne versprengte Trümmer von elastischen Fasern. - Plasmazellen finden sich in spärlicher Zahl in dem Infiltrationswall. Mastzellen noch spärlicher, Riesenzellen überhaupt nicht. Die Schweißdrüsen erscheinen unverändert.

Fall 4. Grete Sp. 17 Jahre alt. Im Haushalte tätig. Familienanamnese o. B.

Die Affektion besteht seit einem halben Jahr; Ursache unbekannt. Frostes.

Status: Pat. leidet an einer starken venösen Stauung an den Händen infolge

Befallen sind die Knöchel des IV. und V. Fingers der linken Hand, des IV. Fingers der rechten Hand. Die Efflorescenzen sind ungefähr einpfennigstälckgroB. Typische serpiginöse Anordnung über den beiden. IV. Fingern. Der periphere Rand ist erhaben und hart; zentral sind die Efflorescenzen eingesunken, der Rand ist weíBlich verfärbt, das Zentrum ist bläulich schimmernd. Die Knöchel des V. Fingers der linken Hand sind mit 5 einzelnen, derben, auf der Höhe ziemlich flachen Knötchen bedeckt, sie werden excidiert. Patientin ist sonst gesund.

3. XII. 1913 bis 28. 10. 1915 Behandlung der Pat. mit Röntgen, Weiß- und Blaulioht der Kromayer schen Quarzlampe und Kohlensäureschnee. Auf Röntgen heilten fast alle Stellen ab; Weiß- und Blaulicht versagten oft. Die 1910 excidierte Stelle rezidivierte 1914, es traten im selben und nächstfolgenden Jahre neue Ef. florescenzen auf dem rechten und linken Handrücken auf; eine Efflorescenz auf der linken Hand erreichte die Größe eines Fünfmarkstückes.

23. XI. 1920 stellt Pat. sich wieder vor. (Sie hat vor 4 Jahren geheiratet, hat keine Kinder und keine Aborte gehabt.) Nach der Entlassung aus der Lichtbehandlung (1915) gingen alle Efflorescenzen zurück und sind keine neuen wiedergekommen. Jetzt ist von der früheren Krankheit nichts mehr zu sehen; auch sonst ist Pat. gesund.

Histologischer Befund: Die Übersicht mit schwacher Vergrößerung zeigt in dem Bindegewebe der Cutis drei voneinander durch breite Zonen anscheinend normalen Bindegewebes getrennte, große nekrotische Herde, von denen jeder von einem Wall dichten zelligen Infiltrates rings umgeben ist. Ein solcher be: sonders typisoher Granuloma-annulare-Herd ist in Abb. 1 wiedergegeben. Im einzelnen sind sie von derselben Beschaffenheit wie im Fall 3, so daß sich eine ausführlichere Beschreibung wohl erübrigt und nur kurz zu erwähnen wäre, was der vorliegende Fall außerdem noch bietet. - Die Epidermis zeigt neben ausgesprochener Akanthose eine deutliche Verlängerung der Retezapfen, aber keine Parakeratose. In der etwas ödematösen subpapillaren Schicht sind die fixen Bindegewebszellen erheblich vermehrt. Der Infiltrationswall um die nekrotischen Bindegewebspartien herum zeigt den bereits bekannten Aufbau von lymphocytären und epitheloiden Zellelementen. Die ausgedehnten Nekroseherde lassen keine Strukturverhältnisse mehr erkennen, sondern weisen nur reichliche Kerntrümmer auf und sínd zum Teil verhältnismäßig zahlreich mit polynucleären Leukocyten durchsetzt. Stellenweise finden sich am Rande der nekrotischen Partien Anhäufungen von Fibro- 
blasten. AuBerhalb der untergegangenen Bindegewebspartien ist das kollagene Gewebe überall wohl erhalten und normal gefärbt, ebenso die elastischen Fasern. Die perivasculäre Infiltration ist in besonders ausgesprochenem Maße an allen Gefäßen vorhanden, und zwar auch noch bei den Gefäßen, die schon recht weit von den Haupterkrankungsherden entfernt in anscheinend sonst gesundem Gewebe liegen. Die Gefäße sind in den großen Infiltraten selbst vielfach komprimiert, in der Peripherie derselben aber vielfach erweitert. Veränderungen an der Gefäßwand sieht man nur an einer Stelle, die allerdings ein recht bemerkenswertes Bild gibt. Es schließt sich nämlich an ein durch Endothelwucherung verschlossenes Gefäß ein Nekrosenherd an, bzw. das Gefäß mündet anscheinend in diesen hinein, so da $B$ man den Eindruck erhält, als wenn die Nekrose, die in ihrer Ausdehnung etwa dem Versorgungsbereich des betreffenden verschlossenen Gefäßes entsprechen

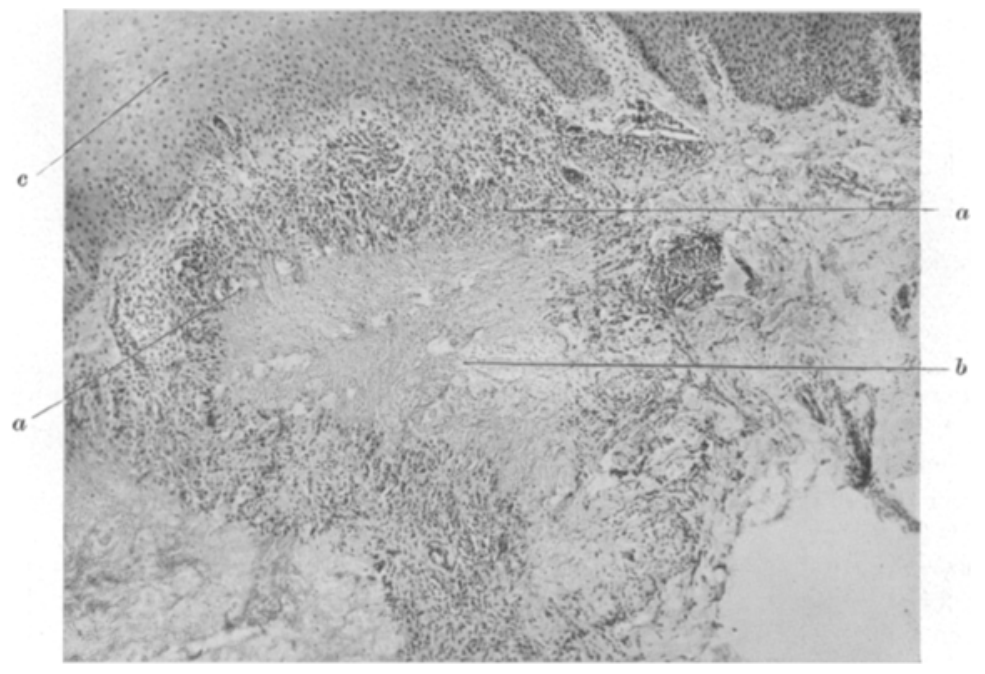

Abb. 1. Typischer Granuloma-annulare-Herd, $a=$ Peripherer Infiltrationswall, $b=$ zentraler Nekroseherd, $c=$ Epidermis.

könnte, die Folge des Gefäßverschlusses wäre. - Stellenweise sind auch die Schweißdrüsen von kleinzelligem Infiltrat umgeben, die Drüsen selbst scheinen unverändert. Plasmazellen sind in den Infiltraten in mäßiger Zahl vorhanden, Mastzellen nicht, dagegen wurden in einem Epitheloidzellhaufen Riesenzellen festgestellt.

Fall 5. Paul Th. 23 Jahre alt, Dreher.

25. 9. 1916. Moulage der Kieler Hautklinik.

Auf dem rechten Handrücken zwischen Daumen und Mittelfinger ein serpiginöser fast ringförmig angeordneter Wall von ca. $1 / 2 \mathrm{~cm}$ Höhe und $3 \mathrm{~mm}$ Breite von weißlicher Farbe. Das Zentrum ist deutlich eingesunken, von bläulich-roter Farbe. In der Peripherie heben sich deutlich einige einzelne Knötchen ab. Excision vom Handrücken. WaR

Dieser Fall weicht im histologischen Bilde etwas von den früheren ab. Da die Abweichungen sich vielleicht als fortgeschritteneres Entwicklungs- bzw. Heilungsstadium des Prozesses erklären lassen und uns manches über die Verlaufsweise der Erkrankung auszusagen vermögen, so ist ihre genaue Beschreibung gerechtfertigt. Die Übersicht mit schwacher Vergrößerung ergibt zunächst wiederum 
eine höchst auffallende, räumlich über einen sehr großen Bezirk ausgedehnte perivasculäre kleinzellige Infiltration hauptsächlich der mittleren Cutis, aber auch der höheren und tieferen Schichten. Die Epidermis zeigt geringe Akanthose ohne sonstige Veränderungen. In der Cutis besteht ein ausgedehnter Infiltrationsherd, der schon mit bloßem Auge in den Schnitten erkenntlich ist. Derselbe weist aber nur in einem Abschnitt so komplette Gewebsnekrose auf, wie wir sie von den vorigen Fällen her kennen. In diesem nekrotischen Teil besteht noch eine ziemliche scharfe Abgrenzung von peripherem Zellinfiltrat und Nekrose, während der andere größere Teil des Erkrankungsherdes nux in kleinen Bezirken untergegangenes strukturund kernloses kollagenes Gewebe, dafür aber um so reichlicher und in ziemlich diffuser Ausbreitung zahlreiche junge Bindegewebszellen mit intensiver Kernfärbung und wohlgefärbte, gut strukturierte Bindegewebsfaserzüge enthält. Das

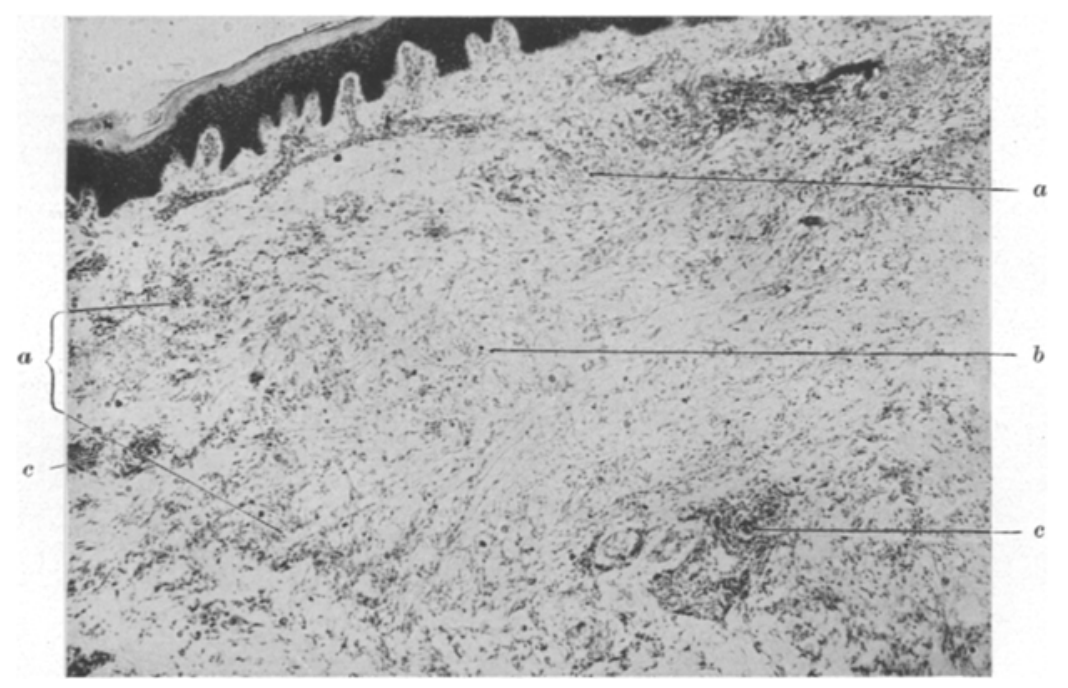

Abb. 2. Granuloma-annulare-Herd in bindegewebiger Umwandlung begriffen. $a=$ Reste des peripheren Infiltrationswalles, $b=$ kernreiches junges Bindegewebe an Stelle der zentralen Nekrose, $c=$ perivaseuläre Infiltration.

Bild, das wix in der Abbildung 2 wiedergeben, erweckt die Yorstellung, als wenn hier an Stelle der nekrotischen Partien größtenteils eine fibroblastische Regeneration Platz gegriffen habe. Auch die kompakte Geschlossenheit des Infiltrationswalles, wie sie in den vorigen. Fällen so charakteristisch in Erscheinung tritt, ist hier nicht vorhanden, das zellige Infiltrat am Rande ist weniger dicht, aufgelockert, als wenn es sich aufzulösen im Begriff wäre. An Stelle der früheren Nekrose, die nur an einzelnen Stellen noch andeutungsweise kenntlich ist, ist ein kernreiches neugebildetes Bindegewebe getreten. Nooh ein zweiter, kleinerer Erkrankungsherd ${ }^{1}$ ) ist in einiger Entfernung von dem eben beschriebenen sichtbar, an dem diese Umwandlungstendenz ebenfalls sehr deutlich sich beobachten läßt. Von dem peripheren Zellinfiltrat her durchsetzen Fibroblasten die nekrotisehe Partie, so daß nur noch in der Mitte ein ganz engex Bezirk von reiner Nekrose vorhanden ist, während im übrigen bereits Bindegewebsneubildungen vorherrschen. Die Veränderungen an den Gefäßen sind hier

1) Der in Abbildung 2 nicht mit enthalten ist. 
etwas ausgeprägter als in den früheren Fällen; es finden sich zum Teil ziemlich starke Endothelwucherungen und typische Bilder von Endarteriitis an kleineren und größeren Gefäßen, und zwar manchmal noch in ziemlich weiter Entfernung von den eigentlichen Infiltraten. Die Venen sind vielfach erweitert. Auffallend ist ein reichlicher Gehalt an Mastzellen in den Infiltraten, spärlicher sind sie im übrigen intakten Bindegewebe vorhanden. Plasmazellen sind geringer vertreten, Riesenzellen fehlen. - Die elastischen Fasern in dem nekrotischen Teil sind zerstört, im übrigen wohl erhalten.

Fall 6. Martha M. 11 Jahre alt, Maschinistenkind. Auf dem rechten Handrücken zwischen Daumen und Zeigefinger auf der radialen Seite des letzteren ein ovaler, etwas unregelmäßiger Herd von ca. $2 \mathrm{mal} 1 \mathrm{~cm}$ Größe mit erhabener fast geschlossener Randzone und eingesunkenem Zentrum. 12. X. 1916 Excision.

18. XI. 1920. Pat. stellt sich wieder vor. Sie glaubt, daß die Affektion einige Jahre bestanden habe. Nach der Excision sei die Hauterkrankung nicht wieder gekommen. Jetzt ist die Hand frei von dieser Affektion, doch man sieht auf dem rechten Handrücken und Unterarm einige typische Planae verucae. In der Hautfalte über dem 2. Interdigitalgelenk ist eine ca. exbsengroße bewegliche Induration zu fühlen. Sonst ist Pat. gesund, auch Eltern und Geschwister sind gesund. Im Urin kein Zucker, kein Eiweiß.

2. XII. 1920. Pat. stellt sich wieder vor. Status idem. Eine Probeexcision der planen Warzen lehnt Pat. ab.

Die mikroskopische Untersuchung des excidierten Stückes ergab das von den vorher beschriebenen Fällen bereits bekannte Bild einer ausgedehnten perivasculären Infiltration der Cutis und herdförmige von zelligem Infiltrat umgebene Nekrosen. Wir sehen in diesem Falle einen aus drei dicht nebeneinanderliegenden Herden offenbar konfluierten großen Nekroseherd, der allseitig von dem typischen Wall von lymphatischen bzw. epitheloiden Zellen umgeben ist. Die Epidermis darüber ist akanthotisch; die Papillen sind etwas verbreitert, die Papillargefäße von ziemlich starker kleinzelliger Infiltration umgeben. Parakeratose besteht nicht. Der Erkrankungsherd in der Cutis erstreckt sich bis in die Tiefe der Schweißdrüsenknäuel, die ebenfalls von kleinzelligem Infiltrat umgeben sind. Verände: rungen an den Gefäßen finden sich außer in Gefäßerweiterungen auch in der Form von Gefäßverschlüssen infolge von Endothelwucherungen an den kleineren Arterien und Venen. Plasma- and Mastzellen kommen sehr spärlich vor, Riesenzellen nicht. Die elastischen Fasern sind in den nekrotischen Partien größtenteils zerstört, im übrigen Gewebe wohl erhalten und normal färbbar.

Fall 7. Walter Sch. 3 Jahre alt, Arbeiterkind. Persönliche und Familienanamnese bietet nichts Besonderes.

30. VIII. 1920. Die augenblickliche Affektion am Zeigefinger besteht seit ca. $1 / 2$ Jahr, sie ist langsam gewachsen, sie hat die Beweglichkeit des Fingers nicht beeinträchtigt. Die Affektion am Fuß besteht kürzere Zeit.

Status: Kräftig gebauter, mit reichlichem Fettpolster versehener Knabe. An den Lungen ist klinisch und mit Hilfe der Röntgendurchleuchtung nichts auf Tuberkulose Verdächtiges festzustellen. Die Halsdrüsen sind beiderseits etwas vergrößert, von weichlicher Konsistenz. Es besteht eine leichte Angina lacunaris. Sonst ist der Status normal. Blutbild: Erythrocyten 4480 000, Leukocyten 7100 Polynucleäre 59\%, kl. Lymphoeyten 30\%, gr. Lymphocyten 7\%, Eosinophile 3\%, Übergangsformen $1 \%$, WaR. — Sachs-Georgi -

Hautbefund, Die Hände zeigen infolge Erfrierens venöse Stauung recht starken Grades Auf der Beugeseite des linken Zeigefingers im Bereiche des II. und III. Gliedes eine kreisförmige Efflorescenz von Pfennigstückgröße. Die Efflorescenz besteht aus einem peripheren $1 / 2 \mathrm{~cm}$ breitem Wall, der in Form eines 
geschlossenen Kreises sich ringförmig um ein eingesunkenes Zentrum hinzieht. Die wallartige Eıhabenheit fällt nach außen hin zur gesurden Haut mit steilen Rändern ab, zur zentralen Partie ist der Übergang weniger steil, aber noch immer dc utlich ausgesprochen. Die Oberhautfelderung ist im Bertiche der ganzen Efflorescenz verstrichen; die Haut der zentralen Partie ist leicht atrophich. Die Farbe des Zentrums ist rosa, die des Walles weiflich elfenbeinartig. Die Efflorescenz erhebt sich über das Niveau der Haut um ca. $1 / 2 \mathrm{~cm}$; auch das eingesunkene Zentrum liegt anccheinend höher als die umgebende normale Haut. Die Affektion. erstreckt sich tumorartig durch die ganze Haut bis zum Unterhautzellgew $\epsilon$ be, über welchem sie gut verschieblich ist. Die Konsistenz dieses Tumors ist derb, fast wie bei einem Keloid. Jucken, Schmerzen, Druckempfindlichkeit besteht nicht. Das Gelenk ist vollständig frei.

Auf der Streckseite der II. Zehe des rechten Fußes ist eine ähnliche Efflorescenz von kaum Bohnengröße vorharden. Eine zentrale Einserkung ist bei diescm Herd kaum angedeutet. Excision des ganzen Herdes am Fuße.

29. X. 1920. Therapeutich erhielt Pat. bis zum heutigen Tage sechs intramuskuläre Terpentineinspritzungen, durchschnittlich einmal in der Woche. Der Herd am Zeigefinger ist weicher geworden. Der wallartige Rard springt nicht mehr so vor, seine Farbe ist noch heller geworden. An GröBe hat der Herd zugenommen und erreicht beiderseits über die Seitenflächen des Fingers hinweg die Streckseite. weisen.

Dio Excision am Fus ist per primam verheilt; ein Rezidiv ist nicht nachzu-

Excision aus dem Herd des Fingers. Nach den Excisionen heilen die übrigen noch vorhandenen Efflorescenzen merkwürd gerweise rasch ab.

Das histologische Bild gestaltet sich hier, obwohl es im Prinzip mit den vorher beschriebenen Fällen übereinstimmt, im einzelnen doch anders, urd zwar sind die Abweichungen für das Verständnis des Krankheitsprozesses so wichtig, daß eine eingehende Beschreibung nicht unterbleiben kann. Das zuerst excidierte Stück vom Fuß ergibt allerdings nichts Besonderes als wieder das bckannte ausgebreitete kleinzellige Infiltrat um die Gefäße herum und einen Nekroseherd in der Cutis, der von einer aus lymphocytären und epitheloiden Zellen gebildeten Randzone umgeben ist. Die Epidermis ist nur. wenig verändert im Sinne einer Akanthose. Im übrigen bieten diese Schnitte in bezug auf die verschicdenen Zell. typen und Gefäßveränderungen dasselbe Bild wie in den anderen Fällen, so daß. einer weiteren Beschreibung nicht bedarf. Arders steht es mit den Präparaten des vom Finger excidierten Hautstückchens. Hier fällt zunächst eine ganz besonders massige und dichte Infiltration um die Gefäße aller Cutiscchichten ins Auge. Das perivasculäre Infiltrat enthält ziemlich viel Plasmazellen und Mastzellen. Das Bindegewebe weist mehrfach nekrotische Partien auf, die sowohl rein herdförmig als auch strangförmig in Zügen angeordnct sird und stellenwrise eine auffallende Gebundenheit an den Verlauf der Gefäße erk€nnen lassen. Wir sehen an manchen Stellen im GefäBverlauf zurächst eine basophile Degeneration des kollagenen Gewebes in der GefäBumgebung auftreten, die dort liegenden Kerne sind schlecht färbbar und zeigen Anzeichen beginnender Nf krose. Fs sik ht so aus, als wenn eine von der Gefäßwand her wirkende Noxe das umgebende Gewebe geschädigt hätte. - Die Form der anderen herdförmigen Nekrosєn ist baumkronenbzw. pilzförmig; auf einem kurzen breiten Stiel, dessen Lage auffallend der Richtung des zuführenden Gefäßes entspricht, sitzt dann wie eine Baumkrone oder ein Pilz der Hauptteil der Bindegewebsnekrose auf. Der peripherische Wall ist außerordentlich reich an Epitheloiden. Stellenweise ist - besonders gut im van Git son. Präparat erkennbar - die Anhäufung von Fibroblasten besonders hervortretend 
und die Neuproduktion von fibrillärem Bindegewebe an feinen gut färbbaren Bindegewebefasern deutlich nachweisbar. Die Gefäße sind in der weiteren Umgebung der Infiltrate erweitert, in den Infiltraten selbst vielfach thrombosiert. An den kleinen Gefäßen sind spärliche Endothelhyperplasien zu sehen, die vereinzelt auch zu vollständigen Gefäßverschlüssen ausgebildet sind. An manchen Stellen scheinen die Kerne der Gefäßwände selbst in ihrer Färbbarkeit beeinträchtigt. Die elastischen Fasern verhalten sich wie in den übrigen Fällen. - Dis Epidermis zeigt wieder Akanthose, aber sonst keine Besonderheiten.

Um die klinischen Hauptmomente unserer Fälle kurz z u sa m men$\mathrm{z}$ ufassen, so ergibt sich, daß das klinische Bild in allen 7 Fällen wohl charakterisiert und durchweg für das Granuloma annulare, wie es von den deutschen Autoren hauptsächlich von Arndt umschrieben wurde, typisch ist. In allen 7 Fällen ist die Lokalisation auf die Hände bzw. Füße beschränkt, immer waren jugendliche Individuen (das jüngste 3, das älteste 27 Jahre alt) befallen, immer gestaltete sich der Prozeß äußerst chronisch. Die Erscheinungsform war stets annähernd die gleiche, es handelte sich um über das Hautniveau ziemlich stark erhabene, weißliche, derbe Knötchen von etwa Linsengröße und darüber, die meist zu größeren runden oder serpiginösen Herden konfluierten; deren Zentrum im Verhältnis zur erhöhten Randzone eingesunken erschien. Für Lues fanden sich, soweit darauf untersucht wurde, keine Anzeichen vor, ebensowenig für Tuberkulose.

Auch histologisch besteht kein Zweifel an der Einheitlichkeit unserer Krankheitsbilder. Soweit sich Unterschiede in den mikroskopischen Befunden ergeben, erklären sie sich wohl aus verschiedenen Stadien des Krankheitsprozesses. Sämtlichen Fällen ist gemeinะam eine höchst auffallende perivasculäre Infiltration aller Cutisschichten, sodann aus lymphocytären und epitheloiden Zellen bestehende sehr große Infiltrate in der Cutis, die in der Form einer peripheren Zone um ausgedehnte zentrale Nekrosen herum angeordnet sind. Mast- und Plasmazellen kommen vor, Riesenzellen nur äußerst selten, wir fanden einmal an einer einzigen Stelle welche. Ferner bestehen fast überall Gefäßveränderungen in der Form von mehr oder weniger hochgradigen Endothelwucherungen an Venen und Arterien. - Die Epidermis ist nur in geringem Grade an dem Prozeß beteiligt, es finden sich immer Akanthosen, einmal auch eine ausgesprochene Parakeratose. - Bakterien sahen wir in den Schnitten nicht; auf Tuberkelbacillen ist allerdings nicht gefärbt worden, weil es uns von vornherein ganz unwahrscheinlich erschien, daß es sich um eine tuberkulöse Ätiologie handeln könnte. Die negativen Ergebnisse anderer Autoren (Arndt u. a.), die daraufhin Untersuchungen anstellten, sprechen ja auch in diesem Sinne. - Im übrigen haben wir natürlich immer Serienschnitte angelegt, um den mikroskopischen Befund vollständig zu erfassen.

Auf Grund unserer histologischen Untersuchungen haben wir uns 
folgende Vorstellung, die sich zum Teil mit der Auffassung von Dalla Favera, Ca pelli u. a. deckt, von dem Wesen der Krankheit gebildet. Das Primäre ist offenbar eine an den Gefäßen der Cutis angreifende Noxe bakterieller oder chemischer, zur Zeit jedenfalls völlig unbekannter Art, die eine proliferative Entzündung des Gefäßendothels und die kleinzelligen Infiltrate, vielleicht auch manchmal direkte toxische Bindegewebsschädigung in der Umgebung der Gefäße verursacht, für welch letztere Annahme sich Anhaltspunkte in unserem Fall 7 finden lassen. Durch endarteriitische Gefäßverschlüsse, die wir nachweisen konnten, kommt es dann wohl zu herdförmigen Ernährungsstörungen im Bindegewebe, welche man direkt als Infarkte auffassen kann, in denen in weiterer Folge eine regressive Metamorphose bis zu völliger Nekrose des Gewebes eintritt. Das stets vorhandene, in ringförmiger Randzone die Nekrosenherde umgebende zellige Infiltrat, das nach dem Zentrum zu vielfach ganz scharf abgesetzt erscheint und dort überwiegend aus epitheloiden Zellen, nach der Peripherie hingegen meist aus Lymphocyten besteht, fassen wir auf als den Ausdruck eines Demarkationsvorganges und eines gleichzeitig vom Rand her sich einleitenden Regenerationsprozesses des untergegangenen Bindegewebes. Dafür sprechen eindeutig die an allen unseren Präparaten beobachteten Neubildungen von frischem fibrillärem Bindegewebe.

Je nachdem, wieweit der Prozeß gediehen ist, kann die histologische Untersuchung in verschiedenen Fällen dann verschiedene Bilder ergeben. Auf diese Weise erklären sich wohl die Unterschiede in den Befunden mancher Autoren. So hat z. B. Vignolo-Lutati in seinem 1912 beschriebenen Falle offenbar einen noch nicht sehr fortgeschrittenen Prozeß vor sich gehabt, denn er spricht nur von chronisch entzündlichen Vorgängen, in denen keine Nekrosen vorhanden waren; vielleicht war es in dem Falle noch nicht zum Verschluß größerer Gefäße und folglich noch zu keiner herdförmigen Nekrosenbildung gekommen.

Andererseits hat Define offenbar einen Fall gesehen, in dem entzündliche Infiltration und Nekrose auf dem Höhepunkt der Ausbildung waren (er spricht von ,myxomatöser Degeneration" und „Koagulationsnekrose "), während er fibroblastische Tätigkeit vermißte.

In dem von Arndt beschriebenen Falle scheint das regenerative Moment mehr im Vordergrunde gestanden und zu der Auffassung dieses Autors von dem Wesen des Prozesses als eines infektiösen Granuloms mit beigetragen zu haben.

In ausgebildeten Fällen von Granuloma annulare ist jedenfalls nach unserer Erfahrung das histologische Bild immer sehr typisch und, wie unsere Beispiele zeigen, so charakteristisch, daß es im Zweifelsfalle zur eindeutigen Entscheidung der klinischen Diagnose wird dienen können. 
Auf eine Diskussion der klinischen und histologischen Differentialdiagnose des Granuloma annulare glauben wir im Interesse der Raumersparnis verzichten zu können, da sie sich in ausführlicher Form in den Arbeiten von Arndt, Vignola-Lutati und a. a. Orten findet.

\section{Literatur.}

Die bis 1916 erschienene Literatur ist bei Liebreich, ,Zur Kenntnis des Granuloma annulare und seiner evtl. Beziehung zum Lichen ruber planus“, Archiv 123, 5. 120. 1916 aufgezählt. Arning, Ein Fall von Granuloma annulare, Derm. Ges. Hamburg. Sitzung v. 5. IV. 1919. Dermatol. Wochenschr. 24, 380. 1919. Hartzell, Bericht über 5 Fälle. Journ. of the Amer. med. assoc. 1914, Juli 1915, S. 230. Arch 122, 951. 1915. - Kiss me yer. Ein Fall von Granuloma annulare. Sitz. d. Dän. Derm. Des. v. 4. X. 1916. Dermatol. Zeitschr. 1919., S. 41. - Little, Granuloma annulare. Royal Soc. of Med. Derm. Abt. Sitz. v. 21. X. 1915. Arch. 122. S. 832. 1915. - Little, Ein Fall von Granuloma annulare. Royal Soe. of med. Derm. Abt. Sitz. v. 16. XII. 1915. Dermatol. Wochenschr. 1916, S. 948. - Sa. c harow, Ein Fall von Granuloma annulare. Russ. Monatsschr. 4, Nr. 10. 1914. Derm. Wochenschr. 1918. S. 778. 